\title{
Symmetric products, linear representations and trace identities
}

\section{Francesco Vaccarino ${ }^{1}$}

Received: 12 May 2020 / Accepted: 23 March 2021 / Published online: 9 April 2021

(c) The Author(s) 2021

\begin{abstract}
We give the equations of the $n$-th symmetric product $X^{n} / S_{n}$ of a flat affine scheme $X=$ Spec $A$ over a commutative ring $F$. As a consequence, we find a closed immersion into the coarse moduli space parameterizing $n$-dimensional linear representations of $A$. This is done by exhibiting an isomorphism between the ring of symmetric tensors over $A$ and the ring generated by the coefficients of the characteristic polynomial of polynomials in commuting generic matrices giving representations of $A$. Using this we derive an isomorphism of the associated reduced schemes over an infinite field. When the characteristic is zero we show that this isomorphism is an isomorphism of schemes and we express it in term of traces.
\end{abstract}

Keywords Symmetric products - Symmetric tensors - Linear representations · Moduli spaces · Determinants · Traces

Mathematics Subject Classification 14L30 - 13A50 - 20G05

\section{Introduction}

Let $F$ be an infinite field and let $F\left[y_{1}, \ldots, y_{n}\right]^{S_{n}}$ be the ring of symmetric polynomials in $n$ variables (see Macdonald 1995). The general linear group $\operatorname{GL}(n, F)$ acts by conjugation on the full $\operatorname{ring} \operatorname{Mat}(n, F)$ of $n \times n$ matrices over $F$. Denote by $F[\operatorname{Mat}(n, F)]^{\mathrm{GL}(n, F)}$ the ring of the polynomial invariants for this actions. It is well known that

$$
F[\operatorname{Mat}(n, F)]^{\mathrm{GL}(n, F)} \cong F\left[y_{1}, \ldots, y_{n}\right]^{S_{n}}
$$

$凶$ Francesco Vaccarino

francesco.vaccarino@polito.it

1 Dipartimento di Scienze Matematiche, Politecnico di Torino, C.so Duca degli Abruzzi 24, 10129 Turin, Italy 
The above result can be restated by saying that the scheme parameterizing $n$ dimensional linear representations of $F[x]$ up to basis change is isomorphic to the symmetric product

$$
\left(\mathbb{A}^{1}\right)^{n} / S_{n}=\operatorname{Spec} F\left[y_{1}, \ldots, y_{n}\right]^{S_{n}}
$$

Let $F$ be a commutative ring. We write $\mathcal{R}_{n}(A) / / \operatorname{GL}(n, F)$ for the coarse moduli space that parameterizes $n$-dimensional linear representations of a flat commutative $F$-algebra $A$ up to basis change. Let $X=\operatorname{Spec} A$ and write $X^{n} / S_{n}$ for the symmetric product of its spectrum.

When $A$ is flat as $F$-module we have a couple of morphisms relating $X^{n} / S_{n}$ to $\mathcal{R}_{n}(A) / / \operatorname{GL}(n, F)$, one is given by the composition of a representation $\rho$ with the usual determinant, the other is induced by the restriction to diagonal matrices.

Our main results are the following generalizations of (2)

- we give a closed embedding of schemes

$$
X^{n} / S_{n} \hookrightarrow \mathcal{R}_{n}(A) / / \operatorname{GL}(n, F)
$$

- when $F$ is an infinite field the embedding (3) induces an isomorphism of the associated reduced schemes.

- when $F$ is a characteristic zero field the embedding (3) is an isomorphism.

Suppose $A$ is generated by say $m$ elements, then one can identify the geometric points of the symmetric product with a sub-scheme of the $m$-tuples of diagonal $n \times n$ matrices and the above result can be read as a theorem of simultaneous diagonalization as well as a generalization of the classical result due to Junker (1893) and Weyl (1946).

As a complement, we give a presentation by generators and relations of $\left(A^{\otimes n}\right)^{S_{n}}$ that holds for $A$ flat and over any commutative base $\operatorname{ring} F$ giving then the equations of $X^{n} / S_{n}$ in the flat case. In the characteristic zero cases, we describe $V_{n}(A)^{\mathrm{GL}(n, F)}$ in terms of traces and polynomial identities.

These results generalize those one given in Vaccarino (2005, 2007). See Vaccarino (2012) for further readings on these topics.

\section{Notation}

- $F$ is a fixed commutative base ring

- we write algebra to mean commutative $F$-algebra

- we denote by $\mathcal{C}_{F}$ the category of commutative $F$-algebras

- Sets the category of sets

- we write $\mathcal{A}(B, C):=\operatorname{Hom}_{\mathcal{A}}(B, C)$ in a category $\mathcal{A}$ with $B, C$ objects in $\mathcal{A}$.

- for $A$ a set and any additive monoid $M$, we denote by $M^{(A)}$ the set of functions $f: A \rightarrow M$ with finite support.

- let $\alpha \in M^{(A)}$, we denote by $|\alpha|$ the (finite) sum $\sum_{a \in A} \alpha(a)$,

- given a set $I$ we denote by $\sharp I$ its cardinality. 


\section{Symmetric product}

Let $A$ be an algebra and $X=\operatorname{Spec} A$ its prime spectrum. The symmetric group $S_{n}$ acts on the $n$-th tensor power $A^{\otimes n}$ and as usual we write $\operatorname{TS}_{F}^{n}(A)$ or simply $\operatorname{TS}^{n}(A)$ to denote the subalgebra of the invariants for this action, i.e. the symmetric tensors of order $n$ over $A$. The $n$-th symmetric product of the affine scheme $X$ is the quotient scheme of $X^{n}$ with respect to the above action and is usually denoted by $X^{(n)}$. By definition $X^{(n)}=X^{n} / S_{n}=\operatorname{Spec} \operatorname{TS}^{n}(A)$.

\subsection{Polynomial laws}

To link symmetric tensors to linear representations we shall use the determinant so that we are lead to the topic of polynomial laws: we recall the definition of this kind of a map between $F$-modules that generalizes the usual polynomial mapping between free $F$-modules (see Bourbaki 1988; Roby 1963, 1980).

Definition 1 Let $A$ and $B$ be two $F$-modules. A polynomial law $\varphi$ from $A$ to $B$ is a family of mappings $\varphi_{L}: L \otimes_{F} A \longrightarrow L \otimes_{F} B$, with $L \in \mathcal{C}_{F}$ such that the following diagram commutes

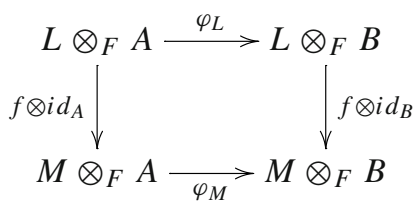

for all $L, M \in \mathcal{C}_{F}$ and all $f \in \mathcal{C}_{F}(L, M)$.

Definition 2 Let $n \in \mathbb{N}$, if $\varphi_{L}(a u)=a^{n} \varphi_{L}(u)$, for all $a \in L, u \in L \otimes_{F} A$ and all $L \in \mathcal{C}_{F}$, then $\varphi$ will be said homogeneous of degree $n$.

Definition 3 If $A$ and $B$ are two $F$-algebras and

$$
\left\{\begin{array}{l}
\varphi_{L}(x y)=\varphi_{L}(x) \varphi_{L}(y) \\
\varphi_{L}\left(1_{L \otimes A}\right)=1_{L \otimes B}
\end{array}\right.
$$

for $L \in \mathcal{C}_{F}$ and for all $x, y \in L \otimes_{F} A$, then $\varphi$ is called multiplicative.

Remark 4 A polynomial law $\varphi: A \rightarrow B$ is a natural transformation $-\otimes_{F} A \rightarrow$ $-\otimes_{F} B$.

Let $A$ and $B$ be two $F$-modules and $\varphi: A \rightarrow B$ be a polynomial law. The following result on polynomial laws is a restatement of Théorème I.1 of Roby (1963).

Theorem 5 Let $S$ be a set and let $a_{s}$ be elements of $A$ such that $a_{s}=0$ except for a finite number of $s \in S$. 
1. Let $L=F\left[x_{s}\right]_{s \in S}$. There exist $\varphi_{\xi}\left(\left(a_{s}\right)\right) \in B$, with $\xi \in \mathbb{N}^{(S)}$, such that:

$$
\varphi_{L}\left(\sum_{s \in S} x_{S} \otimes a_{S}\right)=\sum_{\xi \in \mathbb{N}(S)} x^{\xi} \otimes \varphi_{\xi}\left(\left(a_{S}\right)\right)
$$

where $x^{\xi}=\prod_{s \in S} x_{s}^{\xi_{s}}$.

2. Let $R$ be any commutative $F$-algebra and let $r_{s} \in R$ for $s \in S$, then:

$$
\varphi_{R}\left(\sum_{s \in S} r_{s} \otimes a_{S}\right)=\sum_{\xi \in \mathbb{N}^{(S)}} r^{\xi} \otimes \varphi_{\xi}\left(\left(a_{S}\right)\right)
$$

where $r^{\xi}=\prod_{s \in S} r_{s}^{\xi_{s}}$

3. If $\varphi$ is homogeneous of degree $n$, then one has $\varphi_{\xi}\left(\left(a_{s}\right)\right)=0$ if $|\xi|$ is different from n. That is:

$$
\varphi_{R}\left(\sum_{s \in S} r_{s} \otimes a_{S}\right)=\sum_{\xi \in \mathbb{N}^{(S)},|\xi|=n} r^{\xi} \otimes \varphi_{\xi}\left(\left(a_{S}\right)\right)
$$

In particular, if $\varphi$ is homogeneous of degree 0 or 1 , then it is constant or linear, respectively.

Remark 6 The above theorem means that a polynomial law $\varphi: A \rightarrow B$ is completely determined by its coefficients $\varphi_{\xi}\left(\left(a_{s}\right)\right)$, with $\xi \in \mathbb{N}^{(S)}$.

Remark 7 If $A$ is a free $F$-module and $\left\{a_{t}: t \in T\right\}$ is a basis of $A$, then $\varphi$ is completely determined by its coefficients $\varphi_{\xi}\left(\left(a_{t}\right)\right)$, with $\xi \in \mathbb{N}^{(T)}$. If also $B$ is a free $F$-module with basis $\left\{b_{u}: u \in U\right\}$, then $\varphi_{\xi}\left(\left(a_{t}\right)\right)=\sum_{u \in U} \lambda_{u}(\xi) b_{u}$. Let $a=\sum_{t \in T} \mu_{t} a_{t} \in A$. Since only a finite number of $\mu_{t}$ and $\lambda_{u}(\xi)$ are different from zero, the following makes sense:

$$
\begin{aligned}
\varphi_{F}(a)=\varphi_{F}\left(\sum_{t \in T} \mu_{t} a_{t}\right)=\sum_{\xi \in \mathbb{N}^{(T)}} \mu^{\xi} \varphi_{\xi}\left(\left(a_{t}\right)\right) & =\sum_{\xi \in \mathbb{N}^{(T)}} \mu^{\xi}\left(\sum_{u \in U} \lambda_{u}(\xi) b_{u}\right) \\
& =\sum_{u \in U}\left(\sum_{\xi \in \mathbb{N}^{(T)}} \lambda_{u}(\xi) \mu^{\xi}\right) b_{u} .
\end{aligned}
$$

Hence, if both $A$ and $B$ are free $F$-modules, a polynomial law $\varphi: A \rightarrow B$ is simply a polynomial map.

Definition 8 Let $A, B \in \mathcal{C}_{F}$ be two algebras we write $P_{n}(A, B)$ for the multiplicative homogeneous polynomial mapping $A \rightarrow B$ of degree $n$.

The assignment $B \rightarrow P_{n}(A, B)$ determines a functor $P_{n}^{A}: \mathcal{C}_{F} \rightarrow$ Sets as one can easily check. 


\subsection{Symmetric products as representing schemes}

From now on $A$ will be a flat algebra. There is an element $\gamma_{n} \in P_{n}\left(A, \operatorname{TS}^{n}(A)\right)$ given by $\gamma_{n}(a)=a^{\otimes n}$ such that the composition $\mathcal{C}_{F}\left(\operatorname{TS}^{n}(A), B\right) \ni \rho \mapsto \rho \cdot \gamma_{n} \in P_{n}(A, B)$ gives an isomorphism

$$
\mathcal{C}_{F}\left(\operatorname{TS}^{n}(A), B\right) \stackrel{\cong}{\rightarrow} P_{n}(A, B),
$$

i.e. the functor $P_{n}^{A}$ is represented by the symmetric product $X^{(n)}$, (see Bourbaki 1988, chap. IV). We call $\gamma_{n}$ the universal mapping of degree $n$.

The proof goes as follows: by Rem. 6 a polynomial law is determined by its coefficients. If $A$ is free then one can extract from the set of coefficients of $\gamma_{n}$ a linear basis of $\operatorname{TS}^{n}(A)$. Then any polynomial law homogeneous of degree $n$ correspond to a specialization of these coefficients of $\gamma_{n}$. The requirement to be multiplicative corresponds to the requirement of the above specialization to be an algebra homomorphism, since $\gamma_{n}$ is obviously multiplicative. Then one applies the good properties of $\operatorname{TS}^{n}$ with respect to inverse limits and gets the desired result for flat algebras, see Lazard (1969).

\subsection{Generators}

Let $a \in A$, there is an algebra homomorphism

$$
\eta_{a}: F\left[x_{1}, \ldots, x_{n}\right]^{S_{n}} \cong \operatorname{TS}^{n}(F[x]) \rightarrow \operatorname{TS}^{n}(A)
$$

induced by the evaluation of $x$ at $a$. We write $e_{i}^{n}(a)=\eta_{a}\left(e_{i}^{n}\right)$ where $e_{i}^{n}$ is the $i$-th elementary symmetric polynomial in $n$ variables. Given and independent variable $t$ we have an induced map $\bar{\eta}_{a}: F[t]\left[x_{1}, \ldots, x_{n}\right]^{S_{n}} \cong \operatorname{TS}^{n}(F[t][x]) \rightarrow \operatorname{TS}^{n}(A[t])$ such that

$$
t^{n}+\sum_{i} e_{i}^{n}(a)=\bar{\eta}_{a}\left(t^{n}+\sum_{i} e_{i}^{n}\right)=\bar{\eta}_{a}\left(\prod_{i}\left(t+x_{i}\right)\right)=(t+a)^{\otimes n}
$$

so that $e_{i}^{n}(a)$ is the orbit sum of $a^{\otimes i} \otimes 1^{\otimes n-i}$. Note that $e_{n}^{n}(a)=a^{\otimes n}=\gamma_{n}(a)$.

Proposition 9 (Generators) Let $A$ be a commutative flat algebra generated by $\left\{a_{i}\right\}_{i \in I}$ then $\operatorname{TS}^{n}(A)$ is generated by $e_{i}^{n}(a)$ where $a=\prod_{i} a_{i}^{\alpha_{i}}$ is such that $\sum_{i} \alpha_{i} \leq$ $\max (n, \sharp I(n-1))$ and the $\left\{\alpha_{i}\right\}_{i \in I}$ are coprime.

Proof In Vaccarino (2005, Theorem 1) we proved the above statement for $A=P$ a free polynomial $F$-algebra. For $A=P / I$ flat the morphism $\operatorname{TS}^{n}(P) \rightarrow \operatorname{TS}^{n}(A)$ is onto and the proposition follows.

Proposition 10 Suppose $F \supset \mathbb{Q}$ with $\mathbb{Q}$ the rational integers and let $A$ be generated by $\left\{a_{i}\right\}_{i \in I}$. In this case $\operatorname{TS}^{n}(A)$ is generated by $e_{1}^{n}(a)$ where $a=\prod_{i} a_{i}^{\alpha_{i}}$ is such that $\sum_{i} \alpha_{i} \leq n$.

Proof It follows from Proposition 9, Newton formulas and Noether's bound. 


\subsection{Relations}

Suppose you have a homomorphism $f: A \rightarrow B$ of algebras. One can check that the kernel of $\operatorname{TS}^{n} f: \operatorname{TS}^{n}(A) \rightarrow \operatorname{TS}^{n}(B)$ is linearly generated by the orbit sums (under $S_{n}$ ) of elements $a_{1} \otimes \cdots \otimes a_{n}$ such that $\exists k \in\{1, \ldots, n\}$ with $a_{k} \in \operatorname{ker} f$. Now any such element can be expressed as a polynomial in the $e_{i}^{n}(a)$ with $a$ varying into the set of monomials in the $a_{j}$ (see Vaccarino 2005, Lemma 1.2 and Corollary 2.3). Thus we have the following.

Proposition 11 Let $f: A \rightarrow B$ be an algebra homomorphism then the kernel of $\operatorname{TS}^{n} f: \operatorname{TS}^{n}(A) \rightarrow \operatorname{TS}^{n}(B)$ is generated as an ideal by the elements $e_{i}^{n}(a)$ with $i=1, \ldots, n$ and $a \in \operatorname{ker} f$.

Corollary 12 Suppose $F \supset \mathbb{Q}$ then $\operatorname{ker}^{n} f$ is generated by $e_{1}^{n}(a)$ with a $\in \operatorname{ker} f$.

Proof It follows from the above Proposition by Newton's formulas.

Let $P_{\Omega}=F\left[x_{\omega}\right]_{\omega \in \Omega}$ be the free polynomial algebra on $\Omega$. We set $P_{\Omega}^{+}$for the augmentation ideal i.e. the kernel of the evaluation $x_{\omega} \mapsto 0$ for all $\omega$.

Consider the polynomial ring $F\left[e_{i, \mu}\right]$ freely generated by the symbols $e_{i, \mu}$ with $i \geq 1$ and $\mu$ that varies in the set of monomials $\prod_{\omega} x_{\omega}^{\alpha_{\omega}}$ having coprime exponents. By Proposition 9 for all $n$ there is a surjective homomorphism $\kappa_{n}: F\left[e_{i, \mu}\right] \rightarrow \operatorname{TS}^{n}\left(P_{\Omega}\right)$ given by $e_{i, \mu} \mapsto e_{i}^{n}(\mu)$ if $i \leq n$ and $e_{i, \mu} \mapsto 0$ for $i>n$.

Given $f \in P_{\Omega}^{+}$we can compute $e_{m}^{n}(f)$, orbit sum under $S_{n}$ of $f^{\otimes m} \otimes 1^{\otimes n-m}$ for all $n \geq m$ (for $n<m$ it is zero), and express it as a polynomial in the $e_{i}^{n}(\mu)$ with $\mu$ monomial with coprime exponents. It has been shown that this expression stabilizes as $n>m$ (see Vaccarino 2005, Proposition 3.4 or Vaccarino 2008, 4.4). Thus we have a well defined polynomial law $\bar{e}_{m}: P_{\Omega}^{+} \rightarrow F\left[e_{i, \mu}\right]$ homogeneous of degree $m$. In Vaccarino (2005) we proved that ker $\kappa_{n}$ is linearly generated by the coefficients of $\bar{e}_{m}$ with $m>n$.

We give here an improvement of that result.

Theorem 13 (Relations)

1. Let $A=P_{\Omega} / I$ be a flat algebra, then the kernel of the surjection $F\left[e_{i, \mu}\right] \stackrel{\kappa_{n}}{\rightarrow}$ $\operatorname{TS}^{n}\left(P_{\Omega}\right) \rightarrow \operatorname{TS}^{n}(A)$ is linearly generated by the coefficients of $\bar{e}_{m}$ with $m>n$ jointly with the lifting to $F\left[e_{i, \mu}\right]$ of $e_{k}^{n}(g)$ where $k \leq n$ and $g \in I \subset P_{\Omega}$.

2. Suppose $F$ is an infinite field, then the kernel of $\kappa_{n}$ is generated as an ideal by $e_{m}(f)$ with $m>n$ and $f \in P_{\Omega}^{+}$.

3. Suppose $F$ is an infinite field and let $A=P_{\omega} / I$. The kernel of the surjection $F\left[e_{i, \mu}\right] \stackrel{\kappa_{n}}{\rightarrow} \operatorname{TS}^{n}\left(P_{\Omega}\right) \rightarrow \operatorname{TS}^{n}(A)$ is generated as an ideal by $e_{m}(f)$ with $m>n$ and $f \in P_{\Omega}^{+}$plus the lifting to $F\left[e_{i, \mu}\right]$ of the $e_{k}^{n}(g)$ where $k \leq n$ and $g \in I \subset P_{\Omega}$.

Proof 1. It follows from the previous paragraph and Proposition 11.

2. A linear form that is zero on the linear subspace generated by $e_{k}(f)$ with $k>n$ and $f \in P_{\Omega}^{+}$is zero also on the subspace generated by the coefficients as $f$ varies.

3. By $\mathbf{1}$. and 2 . 
Remark 14 Suppose that $A$ is a finitely generated flat algebra so that $A=$ $F\left[x_{1}, \ldots, x_{m}\right] / I$. We have that $\operatorname{TS}^{n}(A)$ is finitely generated and we can substitute in Theorem 13 the ring $F\left[e_{i, \mu}\right]$ with the finitely generated polynomial ring $F\left[e_{i, \mu}\right]$ such that $i=1, \ldots, n$ and the degree of $\mu$ is not greater then $\max (n, m(n-1))$ by Proposition 9. In this sense Theorem 13 gives the equation of $X^{(n)}$ as a closed sub-scheme of $\mathbb{A}^{k}$ with $k$ depending both on $n, m$ and the base ring $F$.

\section{Representations}

Given an algebra $B$ write $\operatorname{Mat}(n, B)$ for the ring of $n \times n$ matrices with entries in $B$.

Definition 15 For a $n$-dimensional representation of $A$ over $B$ one means an algebra homomorphism $A \rightarrow \operatorname{Mat}(n, B)$. We denote by $\mathcal{R}_{n}(A, B)$ the set of these representations. Given any algebras $A, B$ and a homomorphism $\rho: A \rightarrow B$ we write $(\rho)_{n}$ for the induced map $\operatorname{Mat}(n, A) \rightarrow \operatorname{Mat}(n, B)$.

The assignment $B \rightarrow \mathcal{R}_{n}(A, B)$ gives a covariant functor $\mathcal{R}_{n}^{A}: \mathcal{C}_{F} \rightarrow$ Sets as can be easily checked.

Proposition 16 (De Concini et al. 2005, Sect. 1) For all $n \in \mathbb{N}$ and $A \in \mathcal{C}_{F}$ there exist a unique algebra $V_{n}(A)$ and a unique representation $\pi_{n}^{A} \in \mathcal{R}_{n}\left(A, V_{n}(A)\right)$ such that the map $\rho \mapsto(\rho)_{n} \cdot \pi_{n}^{A}$ is an isomorphism

$$
\mathcal{C}_{F}\left(V_{n}(A), B\right) \stackrel{\cong}{\rightarrow} \mathcal{R}_{n}(A, B)
$$

for all algebra $B$, i.e. the functor from schemes to sets associated to $\mathcal{R}_{n}^{A}$ is represented by the affine scheme $\mathcal{R}_{n}(A)=\operatorname{Spec} V_{n}(A)$

Proof Let $\Omega$ be a set and consider $F\left[\xi_{i j, \omega}\right]$, a polynomial ring where $i, j=1, \ldots, n$ and $\omega \in \Omega$.

Let $A_{\Omega}=F\left\{x_{\omega}\right\}_{\omega \in \Omega}$ be the free associative algebra on $\Omega$ then

$$
\mathcal{R}_{n}\left(A_{\Omega}, B\right) \cong \operatorname{Mat}(n, B)^{\Omega} \cong \mathcal{C}_{F}\left(F\left[\xi_{i j, \omega}\right], B\right)
$$

for any $B \in \mathcal{C}_{F}$. More precisely write $D=\operatorname{Mat}\left(n, F\left[\xi_{i j, \omega}\right]\right)$ and let $\xi_{\omega} \in D$ be given by $\left(\xi_{\omega}\right)_{i j}=\xi_{i j, \omega}, \forall i, j, \omega$, these are called the $(n \times n)$ generic matrices and were introduced by C. Procesi (see Procesi 1967) in this setting.

Let $\pi: A_{\Omega} \rightarrow D$ be the $n$-dimensional representation given by $x_{\omega} \mapsto \xi_{\omega}$. Given then any $\rho \in \mathcal{R}_{n}\left(A_{\Omega}, B\right)$, with $B \in \mathcal{C}_{F}$ there is a unique $\bar{\rho} \in \mathcal{C}_{F}\left(F\left[\xi_{i j, \omega}\right], B\right)$ given by $\xi_{i j, h} \mapsto\left(\rho\left(x_{\omega}\right)\right)_{i j}$ and it is such that the following diagram commutes

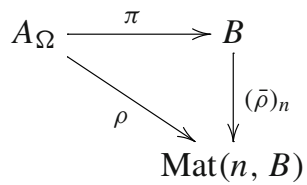


We now substitute $A_{\Omega}$ with an algebra $A \in \mathcal{C}_{F}$ and we let

$$
0 \longrightarrow J \longrightarrow A_{\Omega} \longrightarrow A \longrightarrow 0
$$

be a presentation by generators and relations.

Let $I$ be the unique ideal in $F\left[\xi_{i j, \omega}\right]$ such that

$$
\operatorname{Mat}(n, I)=D \pi(J) D
$$

then as one can easily check that, for all $B \in \mathcal{C}_{F}$

$$
\mathcal{R}_{n}(R, B) \cong \mathcal{C}_{F}\left(F\left[\xi_{i j, \omega}\right] / I, B\right)
$$

via the lifting to a representation of $F$. Set now $V_{n}(A)=F\left[\xi_{i j, \omega}\right] / I$. Let $\left\{a_{\omega}\right\}_{\omega \in \Omega}$ be a set of generators of $A$, in the same way as above we have a universal representation

$$
\pi_{n}^{A}:\left\{\begin{array}{l}
A \longrightarrow \operatorname{Mat}\left(n, V_{n}(A)\right) \\
r_{\omega} \mapsto \xi_{\omega}^{A}
\end{array}\right.
$$

where $\xi_{\omega}^{A}$ is the image of $\xi_{\omega}$ via the surjection $\operatorname{Mat}\left(n, F\left[\xi_{i j, \omega}\right]\right) \rightarrow \operatorname{Mat}\left(n, V_{n}(A)\right)$.

Remark 17 Note that $\mathcal{R}_{n}(A)$ could be quite complicated, as an example, when $A=$ $\mathbb{C}[x, y]$ we obtain that $\mathcal{R}_{n}(A)$ is the commuting scheme and it is not even known (but conjecturally true) if it is reduced or not, see Gan and Ginzburg (2006) and Vaccarino (2007).

\section{Determinant and isomorphism}

We prove the key result of this paper.

Definition 18 For $B \in \mathcal{C}_{F}$ let det $: \operatorname{Mat}(n, B) \rightarrow B$ denote the usual determinant. Let $\chi_{b}(t) \in B[t]$ denote the polynomial ring in the variable $t$ over $B$, the characteristic polynomial of $b \in \operatorname{Mat}(n, B)$ is defined by

$$
\chi_{b}(t)=\operatorname{det}\left(t I_{n}-b\right)=t^{n}+\sum_{i=1}^{n}(-1)^{i} \psi_{i}^{n}(b) t^{n-i}
$$

where $I_{n}$ denotes the $n \times n$ identity matrix.

Definition 19 The subalgebra of $V_{n}(A)$ generated by the coefficients $\psi_{i}^{n}(b)$ for $b$ that varies in $\pi_{n}^{A}(A)$ will be denoted by $C_{n}(A)$.

Theorem 20 The composition det $\cdot \pi_{n}^{A}$ induces an isomorphism for all flat $A \in \mathcal{C}_{F}$

$$
\delta_{n}^{A}: \operatorname{TS}^{n}(A) \stackrel{\cong}{\longrightarrow} C_{n}(A) .
$$


Proof By Sect. 3.2 there is a unique algebra homomorphism $\delta_{n}^{A}: \operatorname{TS}^{n}(A) \rightarrow C_{n}(A) \subset$ $A_{n}(A)$ such that det $\cdot \pi_{n}^{A}=\delta_{n}^{A} \cdot \gamma_{n}$. We have an identification

$$
1_{F[t]} \otimes \delta_{n}^{A}\left(t \otimes 1_{A}+1 \otimes a\right)^{\otimes n}=\operatorname{det}\left(t \otimes 1_{A}+1 \otimes \pi_{n}^{A}(a)\right)
$$

for all $a \in A$ with $t$ and independent variable. Hence $\delta_{n}^{A}\left(e_{i}^{n}(a)\right)=\psi_{i}^{n}\left(\pi_{n}^{A}(a)\right)$ for all $a \in A$ and by definition of $C_{n}(A)$ and Proposition 9 one has that $\delta_{A}^{n}$ is surjective.

By Sect. 4 the representation $\partial_{n}: A \rightarrow \operatorname{Mat}\left(n, A^{\otimes n}\right)$ given by

$$
\partial_{n}(a)=\left(\begin{array}{cccc}
a \otimes 1^{\otimes n-1} & 0 & \ldots & 0 \\
0 & 1 \otimes a \otimes 1^{n-1} & \ldots & 0 \\
\ldots & \ldots & \ddots & 0 \\
0 & 0 & \ldots & 1^{\otimes n-1} \otimes a
\end{array}\right)
$$

corresponds uniquely to a homomorphism $\overline{\partial_{n}}: V_{n}(A) \rightarrow A^{\otimes n}$ such that $\partial_{n}=\left(\overline{\partial_{n}}\right)_{n}$. $\pi_{n}^{A}$. Observe that $\overline{\partial_{n}} \cdot \operatorname{det} \cdot \pi_{n}^{A}=\operatorname{det} \cdot \partial_{n}(a)=a^{\otimes n}=\gamma_{n}(a)$ hence $\overline{\partial_{n}}\left(\psi_{i}^{n}\left(\pi_{n}^{A}(a)\right)\right)=$ $e_{i}^{n}(a)$ for all $a \in A$ and the theorem follows.

\section{Invariants}

We want to study within the Geometric Invariant Theory framework (Mumford et al. 1994) the equivalence classes of representations of $A$ under basis changes, i.e. under the action of the general linear group $\mathrm{G}=\mathrm{GL}(n, F)$. The right object is the categorical quotient $\mathcal{R}_{n}(A) / / \mathrm{G}=\operatorname{Spec} V_{n}(A)^{\mathrm{G}}$, where, as usual, $V_{n}(A)^{\mathrm{G}}$ denotes the invariants for the G-action induced on $V_{n}(A)$ by basis change on $F^{n}$. Observe that $C_{n}(A) \subset$ $V_{n}(A)^{\mathrm{G}}$ thanks to the invariance of the characteristic polynomial by basis change. We address the interested reader to De Concini et al. (2005) and Procesi (1998).

Theorem 21 The homomorphism $\overline{\partial_{n}}: V_{n}(A) \rightarrow A^{\otimes n}$ corresponding to the representation $\partial_{n}$ given in (20) restrict to a surjection $V_{n}(A)^{\mathrm{G}} \rightarrow \mathrm{TS}^{n}(A)$ for all flat algebra i.e. there is a closed embedding

$$
X^{(n)} \hookrightarrow \mathcal{R}_{n}(A) / / \mathrm{G}
$$

Proof Suppose $A$ is presented as in (14). We have the following commutative diagram

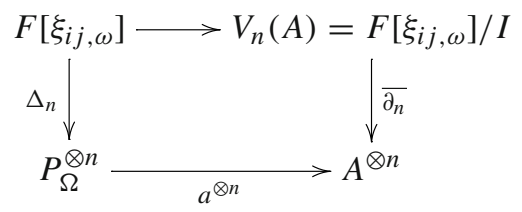


where $\alpha: P_{\Omega} \rightarrow A$ is the surjective homomorphism induced by $A_{\Omega} \rightarrow A$ and

$$
\Delta_{n}\left(\xi_{i j, \omega}\right)= \begin{cases}1^{\otimes i-1} \otimes x_{\omega} \otimes 1^{\otimes n-i} & i=j \\ o & i \neq j\end{cases}
$$

for $i, j=1, \ldots, n$ and for all $\omega \in \Omega$. The symmetric group acts on $V_{n}(A)$ as a subgroup of G. Since $\alpha^{\otimes n}$ and $\Delta_{n}$ are $S_{n}$-equivariant it follows that $\overline{\partial_{n}}$ is $S_{n}$-equivariant too. Thus $\overline{\partial_{n}}: V_{n}(A)^{\mathrm{G}} \rightarrow \mathrm{TS}^{n}(A)$ is onto because $\overline{\partial_{n}} \cdot \delta_{n}^{A}=i d_{\mathrm{TS}^{n}(A)}$ by Theorem 20 .

Remark 22 A classical result due to Junker (1893) and restated by Weyl (1946) says that $\operatorname{TS}^{n} P_{\Omega}$ is generated by the restriction of traces to the diagonal matrices i.e. $F\left[\xi_{i j, \omega}\right]^{\mathrm{G}} \rightarrow \mathrm{TS}^{n} P_{\Omega}$ is surjective over a characteristic zero field $F$. Theorem 21 generalizes Junker - Weyl's result.

Remark 23 Theorem 21 jointly with Theorem 20 says that $\operatorname{Spec} C_{n}(A)$ is identified with a closed sub-scheme of $\mathcal{R}_{n}(A) / / \mathrm{G}$ : those one corresponding to orbits of tuples of diagonal matrices.

\subsection{Infinite field}

In this section $F$ will be an infinite field of arbitrary characteristic. We write $N_{n}(A)$ for the nilradical of $V_{n}(A)$ and $\mathcal{R}_{n}$, red $(A)=\operatorname{Spec} V_{n}(A) / N_{n}(A)$ for the reduced scheme associated to $\mathcal{R}_{n}(A)$. Since $N_{n}(A)$ is G-stable then the action of $\mathrm{G}$ on $\mathcal{R}_{n}(A)$ can be restricted to the variety $\mathcal{R}_{n \text {, red }}(A)$. Thus the homomorphism $\delta_{n}^{A}$ induces another $\delta_{n, \text { red }}^{A}: \operatorname{TS}^{n}(A)_{\text {red }} \rightarrow V_{n}(A)_{\text {red }}^{\mathrm{G}}$

Theorem 24 The homomorphism $\delta_{n, \text { red }}$ is an isomorphism, therefore

$$
X_{\text {red }}^{(n)} \cong \mathcal{R}_{n, \text { red }}(A) / / \mathrm{G}
$$

Proof Let k be the algebraic closure of $F$. Recall that the rational points of GL $(n, F)$ are dense in $\operatorname{GL}(n, k)$ thus we can suppose $F$ algebraically closed without any loss of generality (see Procesi 2007, §6.1). Given a tuple of matrices in $\mathcal{R}_{n}$, red $(A)$ we observe that they are pairwise commuting matrices hence there is $g \in \mathrm{G}$ such that they can be put by $g$ simultaneously in upper triangular form. Consider now a 1-parameter subgroup $\lambda$ of $G$. We choose $\lambda$ such that

$$
\lambda(t)=\left(\begin{array}{cccc}
t^{a_{1}} & 0 & \ldots & 0 \\
0 & t^{a_{2}} & \ldots & 0 \\
\vdots & \vdots & \ddots & \vdots \\
0 & 0 & \ldots & t^{a_{n}}
\end{array}\right)
$$

for some positive integers $a_{1}>a_{2}>\cdots>a_{n}$. 
Let $M$ be an upper triangular $n \times n$ matrix, the map

$$
\lambda: \mathbb{A}^{1} \backslash\{0\} \rightarrow \mathbb{A}^{n^{2}}, t \mapsto \lambda(t) M \lambda(t)^{-1}
$$

can be extended to a regular map

$$
\bar{\lambda}: \mathbb{A}^{1} \rightarrow \mathbb{A}^{n^{2}}
$$

which sends the origin of $\mathbb{A}^{1}$ to the diagonal matrix having the same diagonal elements as $M$. It is clear that the latter belongs to the closure of the orbit of $M$. Thus we have that in the closure of the G-orbit of any point of $\mathcal{R}_{n, \text { red }}(A)$ there is the (closed) orbit of a tuple of diagonal matrices obtained as above. Let now $f \in V_{n}(A)_{\text {red }}^{\mathrm{G}}$ be an invariant regular function that is zero on diagonal matrices. Suppose $f$ is not identically zero, then there is an orbit of a tuple of diagonal matrices over which $f \neq 0$ by continuity. Hence $\delta_{n, \text { red }}^{A}$ is injective. The theorem follows from Theorem 21 .

Remark 25 Let $A=F[x]$ then Theorem 24 implies

$$
F\left[x_{1}, \ldots, x_{m}\right]^{S_{n}} \cong F[\operatorname{Mat}(n, F)]^{\mathrm{G}}
$$

for any infinite field $F$.

\subsection{Characteristic zero}

Theorem 26 Suppose $F$ is a characteristic zero field, then

$$
\delta_{n}^{A}: \operatorname{TS}^{n}(A) \stackrel{\cong}{\rightarrow} V_{n}(A)^{\mathrm{G}}
$$

i.e.

$$
X^{(n)} \cong \mathcal{R}_{n}(A) / / \mathrm{G}
$$

Proof In Vaccarino (2007) we proved that this statement is true when $A$ is a free polynomial ring. The theorem follows because $\mathrm{G}$ is linear reductive (see Mumford et al. 1994, Chapter 1, pag.26) and by Theorem 20.

Another proof. The statement also follows observing that, by Procesi (1976) one has $F\left[\xi_{i j, \omega}\right]^{\mathrm{G}}=C_{n}\left(A_{\Omega}\right)$ and again the result follows by Theorem 20 and the linear reductivity of $\mathrm{G}$.

Remark 27 If $A$ is reduced then Theorem 26 implies that $V_{n}(A)^{\mathrm{G}}$ is reduced too. This gives some support to the conjecture that the commuting scheme is reduced. 


\subsection{Traces}

Along this paragraph we suppose that $n$ ! is invertible in $F$. When we have a polynomial law $\varphi$ homogeneous of degree $n$ we can consider its full polarization $\varphi_{\mathbf{1}_{n}}$ that is the coefficient of $t_{1} t_{2} \ldots t_{n}$ in $\varphi\left(t_{1} x_{1}+\cdots+t_{1} x_{n}\right)$ where $t_{i}$ are commuting independent variables and the $x_{j}$ are generic elements in the domain of $\varphi$. It is well known that the process of polarization is effective via restitution $\varphi_{\mathbf{1}_{n}}(x, x, \ldots, x)=n ! \varphi(x)$.

What happens for $e_{n}^{n}$ ? We write

$$
e_{n}^{n}\left(t_{1} x_{1}+\cdots+t_{n} x_{n}\right)=t_{1} t_{2} \ldots t_{n} e_{\mathbf{1}_{n}}^{n}\left(x_{1}, \ldots, x_{n}\right)+Z
$$

and if one observe that $e_{n}^{n}\left(t_{1} x_{1}+\cdots+t_{n} x_{n}\right)=\left(t_{1} x_{1}+\cdots+t_{n} x_{n}\right)^{\otimes n}$ then it follows easily that $e_{\mathbf{1}_{n}}^{n}\left(x_{1}, \ldots, x_{n}\right)=\sum_{\sigma \in S_{n}} x_{\sigma(1)} \otimes \cdots \otimes x_{\sigma(n)}$. We would like now to express $e_{\mathbf{1}_{n}}^{n}\left(x_{1}, \ldots, x_{n}\right)$ in terms of $e_{1}^{n}(\mu)$ with $\mu$ a monomial in the $x_{i}$.

We know that $\delta_{n}^{A}\left(e_{i}^{n}(a)\right)=\psi_{i}^{n}\left(\pi_{n}^{A}(a)\right)$ where

$$
\operatorname{det}\left(t+\pi_{n}^{A}(a)\right)=t^{n}+\sum_{i} \psi_{i}^{n}\left(\pi_{n}^{A}(a)\right) t^{n-i}
$$

in particular $e_{1}^{n}(a)$ is identified with the trace of $\pi_{n}^{A}(a)$ and $e_{n}^{n}(a)$ with its determinant. It is well known that (see Procesi (1976)) the full polarization $\chi_{\mathbf{1}_{n}}$ of the determinant can be expressed as a special polynomial in traces of monomials. Namely consider the cycle decomposition $\sigma=\sigma_{1} \ldots \sigma_{k} \in S_{n}$ and let correspond to it the product $T_{\sigma}=\operatorname{tr}\left(\mu_{1}\right) \ldots \operatorname{tr}\left(\mu_{k}\right)$ where $\mu_{h}=x_{h_{1}} \ldots x_{h_{l}}$ being $\sigma_{h}=\left(h_{1} h_{2} \ldots h_{l}\right)$, then

$$
\Psi_{\mathbf{1}_{n}}=\sum_{\sigma \in S_{n}} \epsilon_{\sigma} T_{\sigma}
$$

It is a celebrated theorem due to Procesi (1976)) and Razmyslov (1974) that all the relations (in characteristic zero) between the invariants of matrices, i.e. between traces of monomial of generic $n \times n$ matrices, are consequences of $\Psi_{\mathbf{1}_{n+1}}$ in the sense of $T$-ideals.

We summarize what we are able to say so far in the characteristic zero cases.

Theorem 28 Let $A=P_{\Omega} / I$ be a commutative $F$-algebra. The ring of the invariants $V_{n}(A)^{\mathrm{G}}$ is generated by traces of monomial of generic matrices $\xi^{A}$ and the ideal of relations is generated by the evaluation of $\Psi_{1_{n+1}}$ at the elements of $\pi_{n}^{A}(A)$ and by the traces $\operatorname{tr}\left(\pi_{n}^{A}(f)\right)$ with $f \in I$. The same holds mutatis mutandis in $\operatorname{TS}^{n}(A)$

Proof It follows from the preceding paragraph and by Theorem 13.3 using Newton's formulas.

Acknowledgements I would like to thank Michel Brion and Claudio Procesi for useful discussions. Thanks also to the referee for their constructive and helpful comments.

Funding Open access funding provided by Politecnico di Torino within the CRUI-CARE Agreement. 
Open Access This article is licensed under a Creative Commons Attribution 4.0 International License, which permits use, sharing, adaptation, distribution and reproduction in any medium or format, as long as you give appropriate credit to the original author(s) and the source, provide a link to the Creative Commons licence, and indicate if changes were made. The images or other third party material in this article are included in the article's Creative Commons licence, unless indicated otherwise in a credit line to the material. If material is not included in the article's Creative Commons licence and your intended use is not permitted by statutory regulation or exceeds the permitted use, you will need to obtain permission directly from the copyright holder. To view a copy of this licence, visit http://creativecommons.org/licenses/by/4.0/.

\section{References}

Bourbaki, N.: Elements of Mathematics-Algebra II Chapters 4-7. Springer, Berlin (1988)

De Concini, C., Procesi, C., Reshetikhin, N., Rosso, M.: Hopf algebras with trace and representations. Invent. Math. 161, 1-44 (2005)

Gan, W.L., Ginzburg, V.: Almost-commuting variety, D-modules, and Cherednik algebras. IMRP Int. Math. Res. Pap., pp. 1-54 (2006)

Junker, F.: Über symmetrische Functionen von mehreren Reihen von Veränderlichen. Math. Ann. 43, 225270 (1893)

Lazard, D.: Autour de la platitude. Bull. Soc. Math. Fr. 97, 81-128 (1969)

Macdonald, I.G.: Symmetric Functions and Hall Polynomials, 2nd edn. Oxford Mathematical Monograph, Oxford (1995)

Mumford, D., Fogarty, J., Kirwan, F.: Geometric invariant theory, Ergebnisse der Mathematik und ihrer Grenzgebiete 34. Springer, Berlin (1994)

Procesi, C.: Non-commutative affine rings. Atti Accad. Naz. Lincei Rend. Cl. Sci. Fis. Mat. Natur 8, 239-255 (1967)

Procesi, C.: The invariant theory of $n \times n$ matrices. Adv. Math. 19, 306-381 (1976)

Procesi, C.: Deformations of representations. In: Methods in Ring Theory (Levico Terme, 1997), Lecture Notes in Pure and Appl. Math., vol. 198, pp. 247-276. Dekker, New York (1998)

Procesi, C.: Lie groups. An approach through invariants and representations. Universitext Springer, New York (2007)

Razmyslov, J.P.: Identities with trace in full matrix algebras over a field of characteristic zero. Izv. Akad. Nauk SSSR Ser. Mat. 38, 723-756 (1974)

Roby, N.: Lois polynômes et lois formelles en théorie des modules. Ann. Ecole Norm. Sup. 80, 213-348 (1963)

Roby, N.: Lois polynômes multiplicatives universelles. C. R. Math. Acad. Sci. Paris 290, 869-871 (1980)

Vaccarino, F.: The ring of multisymmetric functions. Ann. Inst. Fourier 55, 717-731 (2005)

Vaccarino, F.: Linear representations, symmetric products and the commuting scheme. J. Algebra 317, 634-641 (2007)

Vaccarino, F.: Generalized symmetric functions and invariants of matrices. Math. Z. 260(3), 509-526(2008)

Vaccarino, F.: Moduli of linear representations, symmetric products and the non commutative Hilbert scheme. In: Geometric methods in representation theory II, Séminaires et Congress, Grenoble, vol. 24, pp. 435-456 (2012)

Weyl, H.: The classical groups. Princeton University Press, Princeton (1946)

Publisher's Note Springer Nature remains neutral with regard to jurisdictional claims in published maps and institutional affiliations. 